ARTICLE

\title{
The effect of flow on swimming bacteria controls the initial colonization of curved surfaces
}

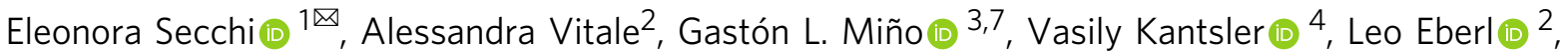 \\ Roberto Rusconi (D) ${ }^{5,6,8 凶}$ \& Roman Stocker (i) ${ }^{1,8 凶}$
}

The colonization of surfaces by bacteria is a widespread phenomenon with consequences on environmental processes and human health. While much is known about the molecular mechanisms of surface colonization, the influence of the physical environment remains poorly understood. Here we show that the colonization of non-planar surfaces by motile bacteria is largely controlled by flow. Using microfluidic experiments with Pseudomonas aeruginosa and Escherichia coli, we demonstrate that the velocity gradients created by a curved surface drive preferential attachment to specific regions of the collecting surface, namely the leeward side of cylinders and immediately downstream of apexes on corrugated surfaces, in stark contrast to where nonmotile cells attach. Attachment location and rate depend on the local hydrodynamics and, as revealed by a mathematical model benchmarked on the observations, on cell morphology and swimming traits. These results highlight the importance of flow on the magnitude and location of bacterial colonization of surfaces.

\footnotetext{
${ }^{1}$ Institute of Environmental Engineering, ETH Zürich, 8093 Zürich, Switzerland. ${ }^{2}$ Department of Plant and Microbial Biology, University of Zürich, 8008 Zürich, Switzerland. ${ }^{3}$ Laboratorio de Microscopía Aplicada a Estudios Moleculares y Celulares (LAMAE), Facultad de Ingeniería, Universidad Nacional de Entre Ríos, 3100 Oro Verde, Argentina. ${ }^{4}$ Department of Physics, University of Warwick, Coventry CV4 7AL, UK. ${ }^{5}$ Department of Biomedical Sciences, Humanitas University, 20090 Pieve Emanuele, MI, Italy. ${ }^{6}$ Humanitas Clinical and Research Center-IRCCS, 20089 Rozzano, MI, Italy. ${ }^{7}$ Present address: Instituto de Investigación y Desarrollo en Bioingeniería y Bioinformática (IBB-UNER-CONICET), 3100 Oro Verde, Argentina. ${ }^{8}$ These authors contributed

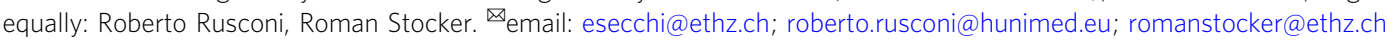


T he attachment of individual bacteria to a surface is the first step toward the formation of surface-attached communities known as biofilms ${ }^{1}$. Biofilms grow on human tissues, including the lungs, urinary tract, eyes, and in chronic wounds ${ }^{2}$, and on implanted devices, such as catheters, prosthetic cardiac valves, and intrauterine devices, posing serious health threats and reducing device lifetime $\mathrm{3}^{3,4}$. Biofouling and biocorrosion are ubiquitous, costly problems also in other settings, from industrial wastewater systems to marine environments ${ }^{5,6}$. To date, the mechanistic understanding of bacterial colonization of surfaces mainly focused on flat surfaces ${ }^{7-12}$; yet in many applications surfaces are not flat. As a result, no general framework exists to account for the role of surface shape on bacterial colonization.

Bacterial transport is often affected by fluid flow, a feature of many microbial habitats. In a straight microfluidic channel, motile bacteria become trapped close to flat surfaces, inducing a strong concentration of cells close to the channel walls ${ }^{12}$. The trapping is a hydrodynamic phenomenon, determined by the action of fluid shear on motile, elongated bacteria ${ }^{12,13}$. Bacterial accumulation also occurs in shallow microfluidic channels behind obstacles and after constrictions ${ }^{14,15}$ and in curved channels downstream of corners, leading to the formation of suspended biofilm structures ${ }^{16-18}$. In groundwater, the size of the grains of the porous matrix ${ }^{19,20}$ and the heterogeneity in flow velocities $^{20,21}$ affect the transport of colloids and, potentially, bacteria. In the human body, secondary flows in the lungs depend on airway geometry ${ }^{22}$, while urine transport is controlled by the amplitude of the contraction waves causing peristaltic motion in the ureter ${ }^{23}$. In the gut, both the luminal flow and the undulated morphology of intestinal villi affect the adhesion to the gut epithelium and the growth of pathogenic bacteria, such as enteroinvasive Escherichia coli, which, in the absence of peristaltic motion, overgrow and trigger an immune response and inflammation $^{24}$. Hosts can also exploit flow-mediated transport of bacteria to favor bacterial adhesion to their surfaces, as occurs in the gut of the bobtail squid Euprymna scolopes, whose cilia create a flow that favors the recruitment of symbiotic Vibrio fischeri ${ }^{25}$. Similarly, the ciliated epidermal surface of corals creates flows that stir the boundary layer, enhancing oxygen transport ${ }^{26}$ and potentially affecting the transport of symbionts and pathogens. In these and many other scenarios, bacteria are recruited to surfaces that are not flat ${ }^{14,15,27-29}$. Yet, despite its biological relevance, a mechanistic understanding of the interplay between flow and bacterial motility in the initial colonization of uneven and curved surfaces is so far missing.

The control of surface contamination by particulates in flowing fluids is important in many applications, such as filtration processes. The microscopic description of filtration usually considers a filter medium as an assembly of collectors, which capture particles suspended in the flow when these encounter one of the collectors $^{30-33}$. A frequent case, also relevant for many microbial processes, is the capture of micron-sized suspended particles by cylindrical collectors in a laminar flow. In this case, the encounter can occur by either direct interception ${ }^{34,35}$, when a particle travels on a streamline that passes sufficiently close (within one particle radius) to the collector to contact it, or diffusional deposition ${ }^{33,35}$, when Brownian motion across the streamline causes the contact. When considering motile microbes rather than passive particles, motility can substantially enhance encounter rates ${ }^{36,37}$, as shown for the colonization of marine organic aggregates ${ }^{38}$. However, encounter rate studies to date have largely neglected the effect of flow on motility. Based on the strong effects of flow on motility observed in straight channels ${ }^{12}$, we asked how flow shapes the encounter rate and encounter location of motile bacteria with curved surfaces.
In this work, we show that the attachment of motile bacteria to curved surfaces is controlled by the effect of the surface on the local flow. Using two model bacteria frequently found in environmental and clinical settings-Pseudomonas aeruginosa and Escherichia coli-we demonstrate that the flow conditions created by the curvature of a surface drive bacteria toward specific locations on the surface. We show that the interplay between local flow and bacterial motility affects both the attachment rate and the attachment site of bacteria, due to the deflection of the trajectories of swimming bacteria by the flow, and that this effect strongly depends on the magnitude of the flow. We present a mathematical model of bacteria swimming in flow, which is in good agreement with the observations and provides a new tool to predict the location and magnitude of bacterial attachment to surfaces.

\section{Results}

Flow affects the attachment rate of bacteria on pillars. As a prototypical case of a curved surface, we first consider the bacterial colonization of cylindrical pillars. This shape represents a simplified model system for grains in porous media ${ }^{39}$, submerged benthic plants and filter fibers ${ }^{35,40,41}$, and tissue heterogeneities in the body such as intravascular pillars ${ }^{42}$. We found that the attachment rate of motile bacteria to a pillar strongly depends on the flow velocity and, in slower flow conditions, can be up to two orders of magnitude larger than for passive particles of the same size. This result was obtained by visualizing GFP-tagged $P$. aeruginosa PA14 swimming near and attaching to single pillars of different diameters $\left(d_{\mathrm{P}}\right)$ exposed to different flow velocities $(U)$ in a microfluidic device (Fig. 1a). Video microscopy was used in fluorescence mode to quantify bacterial attachment to the pillar (Fig. 1d) and in phase-contrast mode to capture bacterial trajectories.

The role of motility was determined by comparing a motile strain (PA14 wt; Fig. 2a, f) with two nonmotile mutants (PA14 $f l g E$; PA14 motB; Fig. 2b, c, f). For the $d_{\mathrm{P}}=100 \mu \mathrm{m}$ pillar, the fluorescent intensity integrated over 5 - $\mu \mathrm{m}$-thick annulus around the perimeter of the pillar, $I_{\mathrm{IN}}$, is more than one order of magnitude higher for motile bacteria than for the two nonmotile mutants at flow velocity $U=300 \mu^{-1}$ (Fig. 2f). This flow velocity is $U / V=6.6$ times the mean bacterial swimming speed $V$ (for PA14 wt, $V=45 \pm 10 \mu \mathrm{m} \mathrm{s}^{-1}$; Supplementary Fig. 1). In the following, we will use the rescaled flow velocity $U / V$ to describe the relative magnitude of the imposed flow. This observation reveals that the strong increase in bacterial capture promoted by motility, already reported for sinking spheres ${ }^{36,37}$, is valid also for pillars, when exposed to moderate flow (up to the threshold of $U / V<20$, determined below). The mechanism of this enhanced capture is known: whereas passive particles and nonmotile bacteria are captured only when the streamline along which they are transported comes within one particle or cell radius from the pillar $^{31,32,35}$, motile bacteria swim across streamlines and can reach the pillar surface from much larger distances ${ }^{36,37}$ (Supplementary Fig. 2).

The motility-induced increase in bacterial accumulation for moderate flow is captured by a mathematical model of bacteria swimming in flow (Fig. 2d, e). In the model, we first computed the three-dimensional velocity field in the microchannel using a finite element code (COMSOL Multiphysics), for the same geometry and flow rate as in the experiments (Fig. 1b, c; Methods). We then used this velocity field to compute the trajectories of single bacteria with an individual-based model (Methods; Supplementary Methods). We modeled bacteria as prolate ellipsoids with aspect ratio $q$ and swimming velocity $V$ directed along the major axis. Their swimming direction at each 


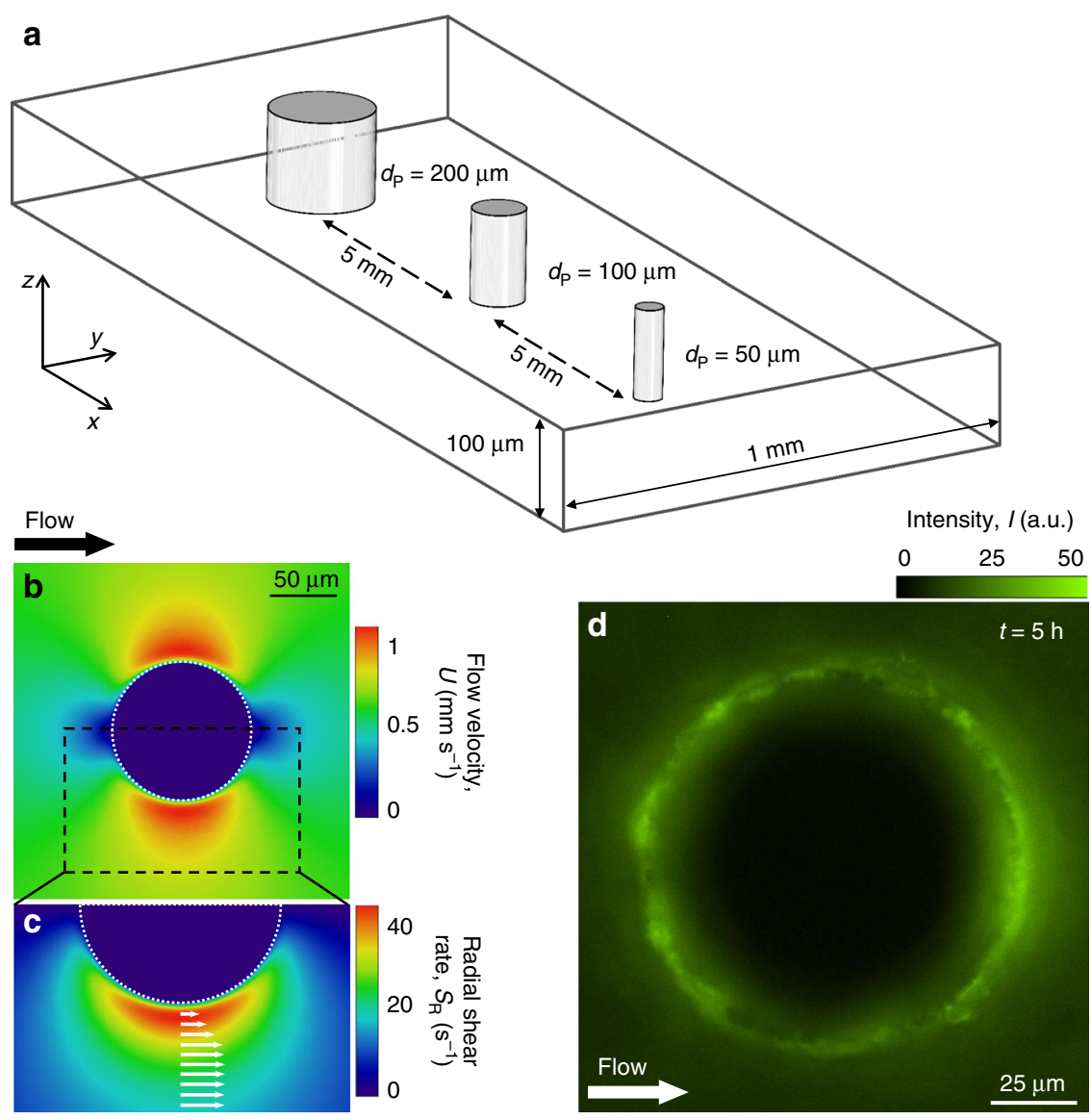

Fig. 1 Microfluidic model of bacterial colonization on curved surfaces in the presence of fluid flow. a Schematic of the microchannel containing pillars of different diameters, $d_{\mathrm{p}}(200 \mu \mathrm{m}, 100 \mu \mathrm{m}$, and $50 \mu \mathrm{m}$; each repeated 2 times). The order of pillars with respect to the flow direction has shown no influence on the observed phenomenon. Flow velocity, $U(\mathbf{b})$ and radial shear rate, $S_{R}(\mathbf{c})$ around a $100-\mu \mathrm{m}$ pillar, computed with COMSOL Multiphysics at a mean flow velocity of $500 \mathrm{um} \mathrm{s}^{-1}$. Superimposed arrows indicate the local velocity field. d Fluorescent image of GFP-tagged P. aeruginosa PA14 wt cells attached to a $100-\mu \mathrm{m}$ pillar after $5 \mathrm{~h}$ flow at rescaled velocity $U / V=3.3\left(U=150 \mu \mathrm{m} \mathrm{s}^{-1}\right)$.

instant in time was determined by a torque balance that accounts for the hydrodynamic shear from the flow and random fluctuations in the bacterial orientation due to rotational Brownian motion or tumbling, which are taken into account using an effective rotational diffusivity ${ }^{12} D_{\mathrm{R}}$. All parameters in the model $\left(q, V, D_{\mathrm{R}}\right)$ were measured directly in separate experiments by tracking individual cells in the absence of flow (Methods; Supplementary Fig. 1). The model scored all contacts with the surface of the pillar (neglecting steric or hydrodynamic interactions) to obtain a capture rate. The capture efficiency, $\eta_{C}{ }^{\text {mod }}$, was computed as the fraction of bacteria removed from the volume of water passing through the region defined by the pillar's cross section, following the classic definition ${ }^{31,35}$. The capture efficiency is equivalent to the capture rate divided by the flux of bacteria passing through the cylinder ${ }^{35}$ and can be directly compared with its experimental counterpart, $\eta_{C}$ exp, which was obtained from the integrated fluorescent intensity $I_{\mathrm{IN}}$ (Methods).

Two regimes emerge when considering the capture efficiency of motile cells as a function of the imposed flow - one regime for moderate flows $(U / V<20)$ and a different regime for strong flows $(U / V>20)$. In the moderate flow regime, the capture efficiency is found to be strongly dependent on fluid velocity, but independent of the pillar diameter: the capture efficiencies $\eta_{C}{ }^{\bmod }$ and $\eta_{C}{ }^{\exp }$ as a function of $U / V$ collapse onto a single curve for different pillar diameters (Fig. 3a, b). This means that for moderate flows, the attachment rate and therefore the density of attached bacteria are the same regardless of pillar diameter (Fig. 3a, b). In this regime, we observe a scaling dependence of both $\eta_{C}{ }^{\bmod }$ and $\eta_{C}{ }^{\exp }$ with $(U / V)^{-1}$ (Fig. 3a, b), indicating that the capture efficiency depends inversely on the fluid velocity (Supplementary Fig. 3a, b) and linearly on the bacterial swimming speed (Supplementary Fig. 3c). In contrast, in the strong flow regime $(U / V>20)$, the predicted capture efficiency $\eta_{C}$ mod does not depend on fluid velocity and decreases with increasing pillar diameter (Fig. 3b). As a result, the attachment density of attached bacteria is higher for smaller pillars, as for the case of passive particles ${ }^{35}$.

The strong increase in the attachment rate of motile compared with nonmotile bacteria can be further seen by considering the capture efficiencies $\eta_{C}{ }^{\text {mod }}$ and $\eta_{C}{ }^{\exp }$ as a function of the Péclet number, $P e=U d_{\mathrm{P}} / D$, which provides a measure of the importance of transport by flow relative to transport by diffusion. Here $D$ is the translational diffusion coefficient of the bacteria, given by the Brownian diffusivity of the cells for nonmotile bacteria or by the effective diffusivity due to motility for motile bacteria, with the latter approximately three orders of magnitude higher than the former ${ }^{43}$ (Supplementary Methods). In our experiments and simulations, motile bacteria are thus characterized by lower values of $P e$ than nonmotile bacteria, given their higher values of $D$. In the low-Pe regime, the enhancement in the capture efficiency is apparent, and can be understood as diffusive transport being important relative to transport by flow, i.e., motile bacteria being able to cross the fluid streamlines owing to their large diffusivity. In this regime, both the experimental and the numerical curve scale as $\sim f\left(d_{\mathrm{P}}\right) P e^{-1.3}$, where $f\left(d_{\mathrm{P}}\right)$ is a 


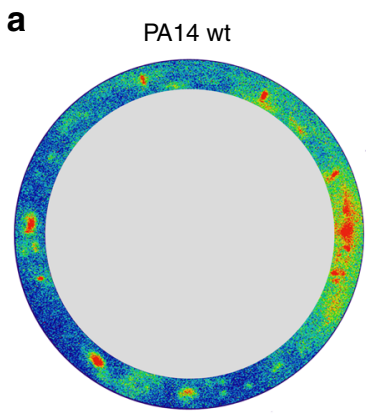

Flow

d

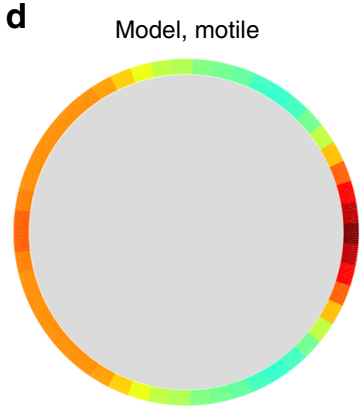

b

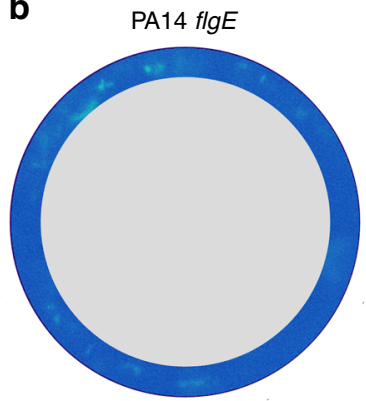

e

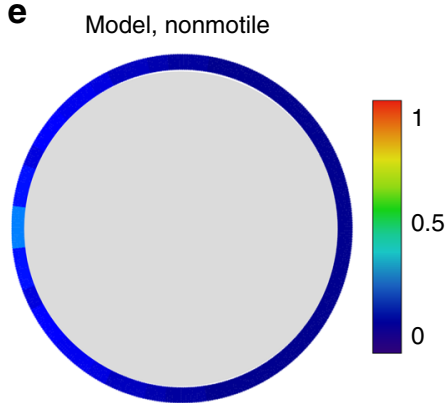

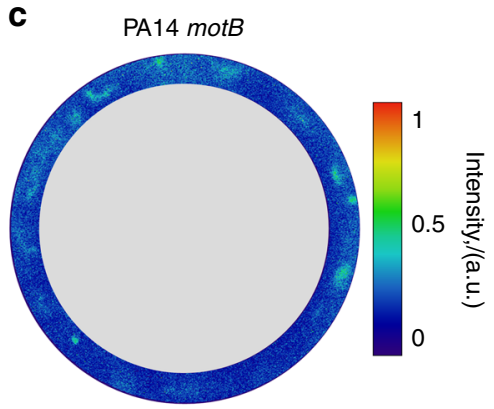

$t=5 \mathrm{~h}$

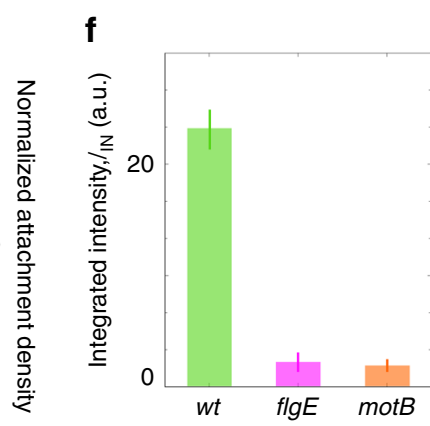

Fig. 2 Surface colonization on pillars by $\mathbf{P}$. aeruginosa is determined by bacterial motility. Intensity distribution of the fluorescent signal from GFP-tagged motile (PA14 wt, a) and nonmotile (PA14 flgE, b; PA14 motB, c) P. aeruginosa cells attached to a 100- $\mu$ m pillar after $5 \mathrm{~h}$ flow at a rescaled flow velocity of $U / V=6.6$. d, e Angular distribution of the normalized attachment density of bacteria on the pillar obtained with a mathematical model for motile (d) and nonmotile (e) cells for the same flow rate and pillar dimension as a-c. $\mathbf{f}$ Integrated intensity, $I_{\mathbb{N}}$ obtained for the motile (wt) and nonmotile (flgE, motB) strains from the images in a-c. Error bars correspond to the standard error of the mean.

coefficient which depends linearly on the pillar diameter (Fig. 3c, d). For increasing flow rate, $P e$ increases and the capture efficiency of motile bacteria rapidly decreases as the role of transport by flow increases over transport by diffusion, until $P e$ is so large (corresponding to the strong flow regime, $U / V>20$ ) that diffusive transport is overcome by transport due to fluid flow (Fig. 3c, d, vertical dotted lines). In the latter regime, attachment rates become comparable with the values for nonmotile bacteria and the capture efficiencies are nearly independent of $P e$ (Fig. 3d). We note that cell shape plays only a modest role in determining the overall capture rate in the regime we investigated (Supplementary Fig. 6j), implying that the overall capture rate is controlled primarily by the swimming speed and not by the local shear rate.

Fluid shear causes leeward adhesion of bacteria to pillars. Motility in flow affects not only the magnitude of attachment but, importantly, also the location of attachment. We demonstrate this first by considering again the case of a cylindrical pillar, both through tracking of individual bacteria before they contact the pillar, and by quantifying the spatial distribution of attachment on the pillar. At flow velocities that are up to a few times the bacterial swimming speed $(U / V \approx 3-6)$, tracking of individual $P$. aeruginosa cells in flow revealed trajectories directed toward the leeward side of the pillar (Fig. 4a, warm colors). These trajectories can be explained in terms of the effect of fluid flow on swimming cells. The no-slip condition on the surface of the pillar creates local velocity gradients (here for brevity termed "shear") (Fig. 1b, c). Shear induces bacteria to rotate with the local angular velocity of the flow ${ }^{44}$. When bacteria are nonmotile, this rotation is rather inconsequential, as they simply follow the flow streamlines (Fig. 4a, blue paths). In contrast, when they are motile, it redirects their trajectory ${ }^{12}$ and causes them to reach the leeward side of the pillar (Fig. 4a, red and yellow paths). Because bacteria are preferentially aligned with streamlines (pointing either upstream or downstream) as they are transported past the pillar, the local shear created by the pillar directs bacteria pointing downstream toward the leeward side of the pillar (Fig. 4a, red and yellow paths) and bacteria pointing upstream away from the pillar.

The mechanism of inward focusing at the hands of the shear profile created by the curved surface is confirmed by our mathematical model. The model further shows that, for conditions mimicking those in the experiments, this mechanism is sufficiently rapid to cause bacteria to contact the pillar surface on its leeward side. An important element here is the velocity of the flow in the wake of the cylinder: once bacterial trajectories cross that region, the major axis of their elongated body will be aligned with the streamlines and thus perpendicular to the pillar surface, either facing upstream or downstream. Consequently, if their distance from the pillar surface is such that their swimming speed is larger than the local flow velocity, the cells oriented upstream will be able to swim toward the surface and encounter the leeward side of the pillar (Fig. 4b, black cells); such upstream swimming has been observed also in close proximity of solid surfaces $^{45}$ and for non-Newtonian fluid flows ${ }^{46}$. We note that this process is not dependent upon sensing or directed motion toward the surface, which were not included in the model: leeward contact is driven purely by the deflection of swimmers' trajectories at the hands of the shear flow profile. Finally, even nonmotile bacteria are also rotating with the local angular velocity of the flow, but this reorientation does not result in a net movement toward the pillar surface (Fig. $4 \mathrm{~b}$, gray cells).

By redirecting cells toward the leeward side of the pillar and consequently increasing the number of local contact events with the surface, the interaction between shear and motility promotes the preferential colonization of specific regions of the pillar's surface. The heterogeneity in surface colonization is confirmed by our observations of cell accumulation around the perimeter of the 
a

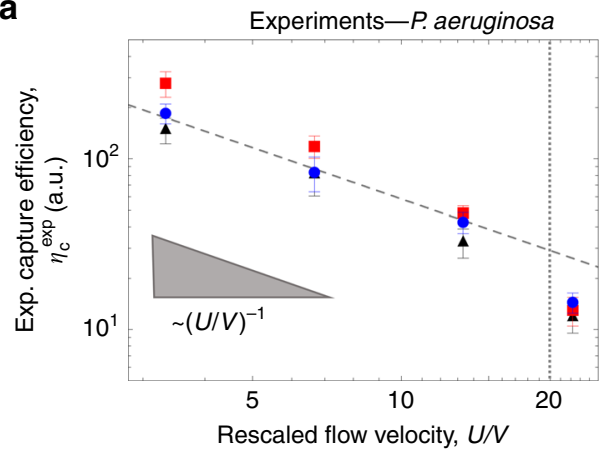

C

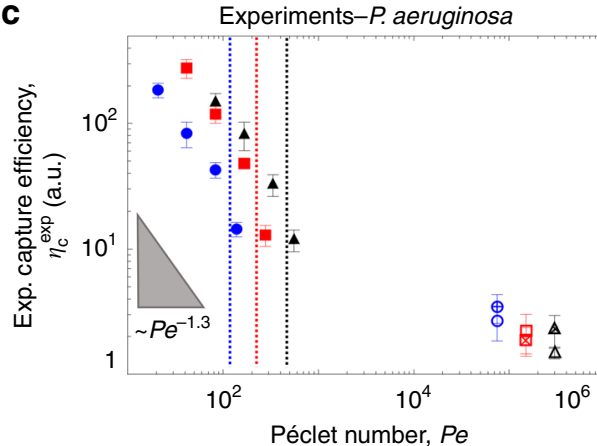

b

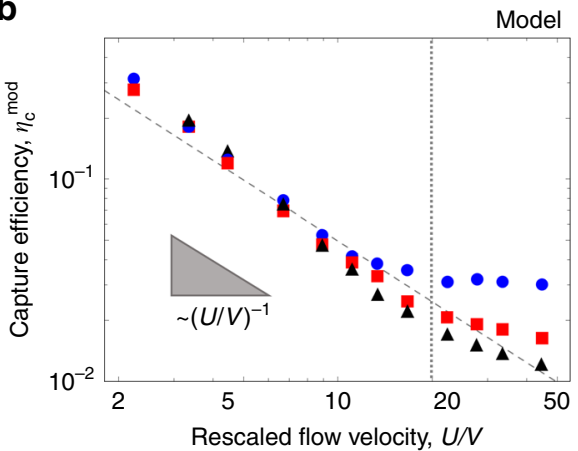

d $\quad$ Model

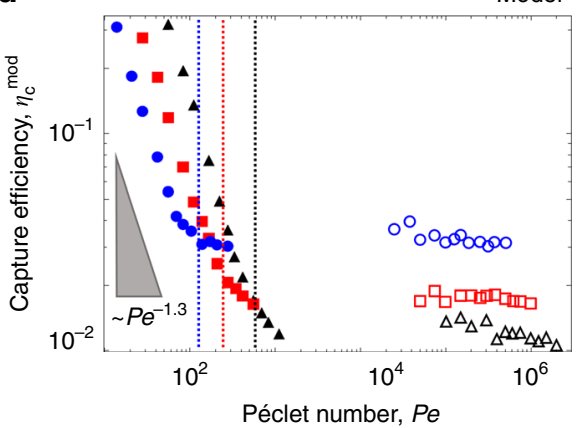

Fig. 3 Capture efficiency of $\boldsymbol{P}$. aeruginosa depends on pillar dimension and flow velocity. a Experimental capture efficiency, $\eta_{C}{ }^{\text {exp }}$, of motile $P$. aeruginosa PA14 wt cells, as a function of the rescaled flow velocity $U / V$, for pillars of diameter $50 \mu \mathrm{m}$ (blue circles), $100 \mu \mathrm{m}$ (red squares) and $200 \mu \mathrm{m}$ (black triangles), measured after $5 \mathrm{~h}$ from the start of the experiment. The dashed curve shows the scaling $\eta_{\mathrm{C}}^{\exp } \sim(U / V)^{-1}$. b Capture efficiency, $\eta_{C}{ }^{\text {mod }}$, as a function of $U / V$, obtained from the model for the same pillar diameters as in (a). c Experimental capture efficiency, $\eta_{C}{ }^{\exp }$, as a function of the Péclet number, Pe, for motile PA14 wt (filled symbols) and nonmotile (PA14 flgE, open symbols with a cross; PA14 motB, open symbols) cells, for pillars of different diameters. $\mathbf{d}$ Capture efficiency, $\eta_{C}{ }^{\text {mod, }}$, as a function of the $P e$, obtained from the model for different pillar diameters, in the case of motile (filled symbols) and nonmotile (open symbols) cells. Vertical dotted lines in $\mathbf{c}$ and $\mathbf{d}$ represent the Pe numbers corresponding to $U / V=20$, calculated for each pillar dimension. Error bars correspond to the standard error of the mean.
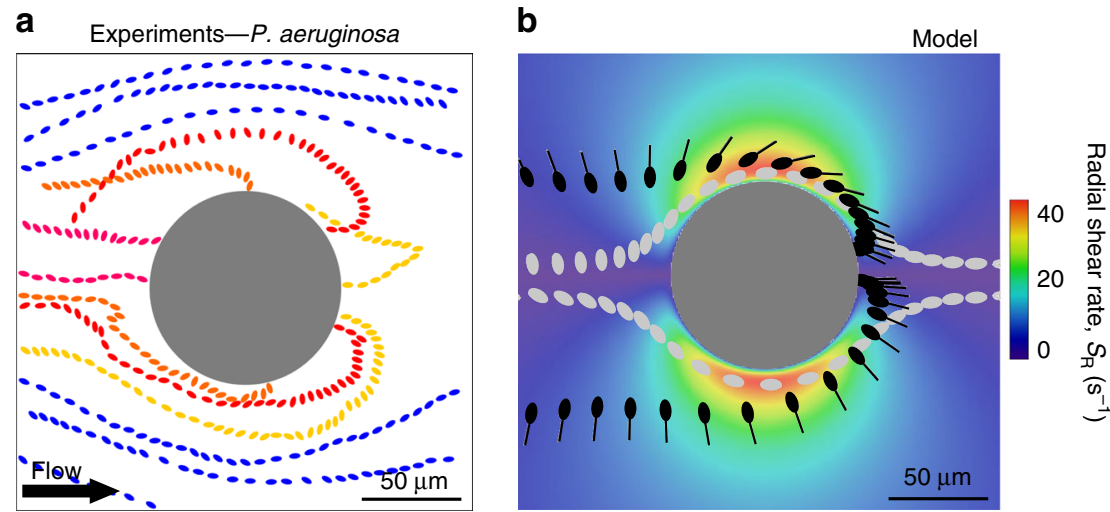

Fig. 4 Fluid shear affects the trajectories of swimming bacteria around a pillar. a Sample trajectories and orientation of $P$. aeruginosa PA14 wt cells in flow around a $100-\mu \mathrm{m}$ pillar at $U / V=3.3\left(U=150 \mu \mathrm{m} \mathrm{s}^{-1}\right)$, for motile (warm colors) and nonmotile (blue) cells. b Trajectories of motile (black) and nonmotile (gray) cells, simulated without rotational noise $\xi_{R}$, in flow around a $100-\mu m$ pillar at $U / V=3.3$ simulated with the model. The color scale represents the radial shear rate, $S_{R}$, around a $100-\mu \mathrm{m}$ pillar at $U=500 \mu \mathrm{m} \mathrm{s}^{-1}$, reported in Fig. 1c.

pillar, measured in terms of the fluorescence intensity of motile, GFP-tagged PA14 wt cells (Fig. 1d; Supplementary Methods). The preferential leeward attachment is clearly visible in terms of a higher fluorescence intensity, for example for the case of a flow velocity $U / V=6.6$ (Fig. 2a). Preferential leeward attachment was observed only for motile cells, and was not observed for the two nonmotile PA14 strains (flgE and motB; Fig. 2b, c). The latter preferentially attach on the windward side of the pillar, as expected for passive particles ${ }^{35}$. The mathematical model shows that bacterial motility determines the distribution of bacterial attachment sites around the pillar: motile cells preferentially attach on the leeward surface of the pillar (orange curve, Supplementary Fig. 4), whereas nonmotile cells attach only on its windward side (yellow curve, Supplementary Fig. 4). Furthermore, in experiments in which the flow direction was reversed after $2.5 \mathrm{~h}$, a second, symmetric attachment peak formed on the opposite side (the originally windward side) of the pillar over the ensuing $2.5 \mathrm{~h}$, independently of the growth medium (Supplementary Fig. 5), further confirming the robustness of the phenomenon. 
a

b

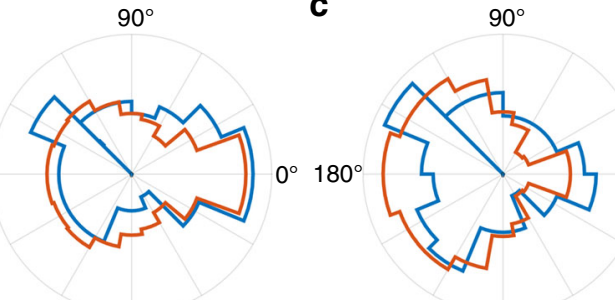

$270^{\circ}$ $270^{\circ}$ d

$90^{\circ}$

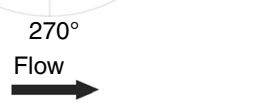

e

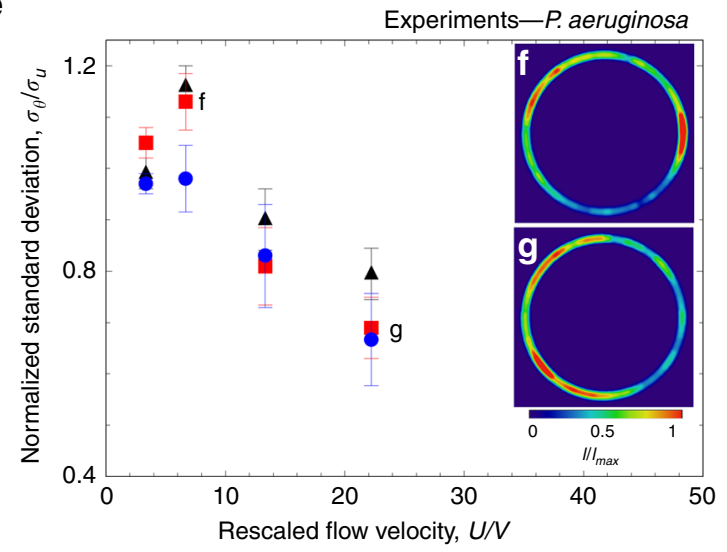

h

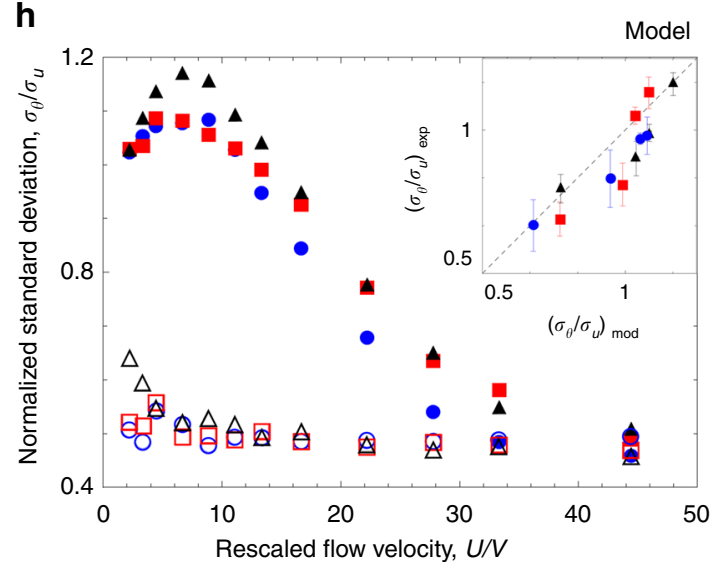

Fig. 5 Fluid velocity modifies the angular distribution of bacterial colonization around a pillar. Angular distribution of the fluorescence intensity, I (blue; experiments after $5 \mathrm{~h}$ of flow of a diluted suspension of PA14 wt GFP cells) and the simulated attachment density (orange; model) on a 100- $\mu$ m pillar for a mean rescaled flow velocity $U / V$ of $3.3(\mathbf{a}), 6.6$ (b), 13.3 (c) and 22.2 (d). e Normalized standard deviation $\sigma_{\theta} / \sigma_{u}$ for motile PA14 wt bacteria as a function of the rescaled flow velocity, $U / V$, for pillars of diameter $50 \mu \mathrm{m}$ (blue circles), $100 \mu \mathrm{m}$ (red squares) and $200 \mu \mathrm{m}$ (black triangles). (Insets) Intensity distribution I of PA14 wt cells, normalized by the maximum value $\left(I_{\max }\right)$, attached to a $100-\mu \mathrm{m}$ pillar at $U / V=6.6(\mathbf{f})$ and $U / V=22.2(\mathbf{g})$. At moderate flows, preferential colonization occurs on the leeward side of the pillar. Flow direction is from left to right. $\mathbf{h}$ Normalized standard deviation $\sigma_{\theta} / \sigma_{u}$ obtained with the model as a function of $U / V$ and for the same pillar dimensions as in $\mathbf{e}$. Results for motile cells (filled symbols) and for nonmotile cells (open symbols) are shown. (Inset) $\sigma_{\theta} / \sigma_{u}$ of the experimental intensity angular distribution as a function of $\sigma_{\theta} / \sigma_{u}$ from the model for different pillar diameters. The dashed line represents $y=x$. Error bars correspond to the standard error of the mean.

The flow velocity affects the distribution of attachment of motile bacteria around the pillar, with higher flow velocities shifting the attachment from the leeward to the windward side. Leeward attachment was observed for motile cells in moderate flows $(U / V<20$; Figs. 2a, $5 \mathrm{f})$. In contrast, for strong flows $(U / V>$ 20 ), motile bacteria attached preferentially to the windward side (Fig. $5 \mathrm{~g}$ ), as passive particles $\mathrm{do}^{35}$. The shift in the location of preferential attachment as a function of the flow velocity can be quantified by computing the angular distribution of the attachment sites on the surface of the pillar. These distributions were obtained from the experiments by using the fluorescence intensity signal of the GFP-tagged bacteria attached along the perimeter of the pillar (blue lines in Fig. 5a-d) and from the model by using the distribution of contact sites obtained from the trajectories of $10^{5}$ bacteria (orange lines in Fig. 5a-d). The angular distributions of bacterial attachment accord in the model and experiments and show a clear dependence on flow velocity. At a low flow velocity of $U / V=3.3$ we observed an accumulation region on the leeward side of the pillar (Fig. 5a), which becomes more pronounced when the flow velocity is doubled $(U / V=6.6$; Fig. 5b, f). A further increase in the flow velocity $(U / V=13.3)$ shifts the preferential attachment toward the windward side of the pillar and leaves just a small leeward peak (Fig. 5c), which completely disappears at $U / V=22.2$ (Fig. $5 \mathrm{~d}$, g). This shift is consistent with the observed transition from a motilitydominated to a flow-dominated regime with increasing flow velocity (Fig. 3).
The change in the distribution of attachment of motile bacteria with flow velocity can be quantified in terms of the standard deviation, $\sigma_{\theta}$, of the angular distribution, normalized by the standard deviation, $\sigma_{u}=\pi / \sqrt{3}$, of a uniform angular distribution $^{41}$. Values of $\sigma_{\theta} / \sigma_{u}>1$ denote a distribution skewed toward the leeward side of the pillar, whereas values of $\sigma_{\theta} / \sigma_{u}<1$ denote preferential colonization of the windward side. This analysis reveals that the skewness of attachment in the leeward direction is most pronounced for $U / V=6.6\left(\sigma_{\theta} / \sigma_{u}>1\right)$ (Fig. 5e, f) and diminishes at $U / V=22.2\left(\sigma_{\theta} / \sigma_{u}=0.6\right)$ (Fig. 5 e, g), as the role of bacterial motility decreases and cells are primarily transported by the flow, akin to passive particles $\left(\sigma_{\theta} / \sigma_{u}=0.37\right)^{41}$. The fact that the passive-particle limit is not reached in the experiments even for the highest flow velocity tested $\left(1000 \mu \mathrm{m} \mathrm{s}^{-1}\right)$ implies that even when the flow speed is substantially higher than the swimming speed $(U / V=22.2)$, the process of attachment is not completely driven by flow, but still influenced by motility. In addition, the model for motile cells (Fig. 5h, filled symbols) shows the transition from leeward to windward attachment as the flow velocity increases from $2.2 \mathrm{~V}$ to $22.2 \mathrm{~V}$, in very good agreement with observations (Inset of Fig. 5), and also reaches the passive direct interception limit $\left(\sigma_{\theta} / \sigma_{u}=0.37\right)$, at a flow velocity $U / V=$ 33.3. In the model, no dependence of $\sigma_{\theta} / \sigma_{u}$ on the flow velocity is observed for nonmotile bacteria (Fig. 5h, open symbols), confirming that the transition from leeward to windward attachment is determined by the interplay between bacterial motility and fluid flow. This transition in the bacterial 
distribution corresponds to the motility-dominated to flowdominated transition observed in the capture efficiency for $U / V>$ 20 (Fig. 3b, d): the interaction of motility and flow thus causes major differences in the capture of motile and nonmotile bacteria not only in terms of the magnitude of bacterial attachment to surfaces, but also in terms of the spatial distribution of attachment.

Cell shape is a key determinant of the inward focusing and leeward encounter process. While self-propulsion is necessary for a cell to reach the pillar surface once in the leeward stagnation region, it is the elongated shape of bacteria that is responsible for their preferential alignment with the flow direction during transport past the cylinder, and hence their inward motion toward the leeward stagnation region. This preferential alignment is well known in the context of Jeffery orbits ${ }^{12,47}$, i.e., the periodic rotation of spheroids in laminar flow: the more elongated the spheroid, the larger the fraction of time it spends aligned with streamlines. The role of elongation is confirmed by results from our model, showing that swimming spherical cells, which reach the leeward stagnation region with a random orientation, have a lower attachment density to the leeward surface of the pillar (Supplementary Fig. 6). We therefore expect that spherical swimmers, such as the alga Chlamydomonas reinhardtii ${ }^{48}$, will not exhibit preferential leeward encounters through this mechanism (Supplementary Fig. 6e-i). The vast majority of motile bacteria, however, are elongated, either because of the elongation of their cell body, or even more so because of the presence of flagella that enhance their effective aspect ratio ${ }^{12}$. Numerical results predict a sharp increase in the leeward attachment when swimmer elongation increases from 1 (spherical) to 3 , and a plateau in the attachment for further increases in elongation (Supplementary Fig. 7i).

A further trait affecting the transport of bacteria toward the pillar is the frequency of the cells' own reorientation (or "tumbling"), which can be expressed in terms of a rotational diffusivity, $D_{\mathrm{R}}{ }^{12}$. Simulations show that cells with low rotational diffusivity $\left(D_{\mathrm{R}}=10^{-2} \mathrm{rad}^{2} \mathrm{~s}^{-1}\right.$; "smooth swimmers") have a stronger tendency for leeward attachment compared with cells with high rotational diffusivity $\left(D_{\mathrm{R}}=1.4 \mathrm{rad}^{2} \mathrm{~s}^{-1}\right.$; value obtained for $P$. aeruginosa, 'tumbling cells', Supplementary Figs. 1, 7e-g). A further (though unrealistically high) increase in rotational diffusivity $\left(D_{\mathrm{R}}=10 \mathrm{rad}^{2} \mathrm{~s}^{-1}\right)$ results in preferential windward attachment (Supplementary Fig. 7h). These results support the hypothesis that alignment along streamlines and reorientation by local shear drive leeward attachment, whereas random fluctuations in the orientation of bacteria such as those caused by tumbling diminish it. Moreover, even for smooth swimmers the model predicts the transition from leeward to windward attachment with increasing flow velocity, but with peaks in the colonization location that are more pronounced compared with those obtained for tumbling cells (Supplementary Fig. 8).

Leeward attachment originates from the interaction between motility and the shear flow profile generated by the pillar, not from the physico-chemical properties of the pillar. We fabricated PDMS pillars of different wettability (Fig. 6a) and stiffness (Fig. 6d), and tested these for a single flow rate $(U / V=6.6$; Fig. 6). Whereas surface coverage varied with surface properties (being lower for more hydrophilic and softer surfaces; Fig. 6c, f), the angular distribution of surface colonization was similar and a leeward attachment peak was robustly observed for all pillars tested (Fig. 6b, e). Similarly, leeward attachment is not a consequence of the use of a specific medium. Growth media can affect bacterial adherence to surfaces, due to changes in $\mathrm{pH}$ and electrolyte concentration ${ }^{49}$. We quantified the attachment distribution of bacteria in two media ( $\mathrm{TB}$ and $\mathrm{AB}$ minimal medium) and for different dilutions of the same medium
(Supplementary Methods). Results show that the bacterial distribution was similar regardless of the medium used and the leeward attachment was always observed (Supplementary Fig. 9). The use of a diluted medium allows us to exclude that bacterial growth was responsible for the observed angular distribution of bacteria around the pillar, as growth in the diluted medium would have been too slow. Taken together, these observations confirm the purely hydrodynamic nature of the leeward attachment phenomenon, which does not depend on the physico-chemical properties of the surface or on the adhesiveness of bacteria. The increase in bacterial density in preferential areas of the pillar is due to a flow-induced increase in the probability of contact between the bacteria and the surface, thus creating a preferential colonization spot.

Shear promotes attachment of bacteria to corrugated surfaces. The flow-induced preferential leeward attachment of motile bacteria to curved surfaces is a more general phenomenon, characterizing attachment on uneven surfaces for different bacterial species. We demonstrate this by showing the spatial attachment pattern of GFP-tagged E. coli (strain MG1655) in a microfluidic channel with sinusoidally shaped sidewalls $(50 \mu \mathrm{m}$ wavelength, $25 \mu \mathrm{m}$ amplitude; Fig. 7a), and extending our mathematical model to this geometrical configuration. Bacterial surface colonization was again quantified in terms of fluorescence intensity in the experiments and contact events with the surface in the model. At moderate flows $\left(U=150 \mu \mathrm{m} \mathrm{s}^{-1} ; U / V=6.9\right.$, calculated using the swimming speed of $E$. coli ${ }^{28}, V=21.6 \mu \mathrm{m} \mathrm{s}^{-1}$ ), bacteria attached to the channel sidewalls preferentially in the region immediately following the apexes of the sinusoid, both in the experiments (Fig. 7b) and the model (Fig. 7c). Preferential attachment leeward of apexes is shown, for example, by the peak in the fluorescent intensity near the location $x=20 \mu \mathrm{m}$ of a period of the sinusoidal surface, obtained experimentally by averaging over 100 identical periods (Fig. 7b) and confirmed by modeling results (Fig. 7c). The mechanism is the same as for pillars: elements of a curved surface that protrude into the flow in this case, the apexes of the sinusoid - generate a locally higher shear (Fig. 7d), which rotates bacteria and drives motile bacteria toward the surface. As a result, they preferentially contact the surface downstream of the protruding element, if their swimming speed is greater than the local flow velocity (Fig. 7e, yellow cell). These results thus suggest that our findings can be generalized: fluid shear promotes the preferential attachment of motile bacteria to the leeward side of curved elements of a surface.

Surface regularity is not a prerequisite of leeward attachment. To illustrate this, we carried out microfluidic experiments with an irregularly corrugated surface (Fig. 8), a model for surface roughness in natural and artificial microbial environments. Experimental observations and modeling results both again show strong heterogeneity in surface colonization by motile bacteria (Fig. 8). The model also shows that this heterogeneity is absent for nonmotile bacteria (Supplementary Fig. 10). Preferential attachment after apexes can be even more pronounced than for the sinusoidal surface, as indicated by both the experimental data (Fig. 8a, b) and the model (Fig. 8c). Examples include the peaks in the fluorescent intensity near the locations $x=450$ and $700 \mu \mathrm{m}$ (Fig. 8). This is in line with the fact that an irregular surface is composed of elements with significantly different curvature, each of which affects the flow (and thus the shear profile) differently. Despite the topographical complexity of this surface, the model also predicts strong preferential attachment locations, with reasonable agreement with the observations. The complexity of this configuration, which we suggest partly accounts for the differences between observations and model, stems from the fact 

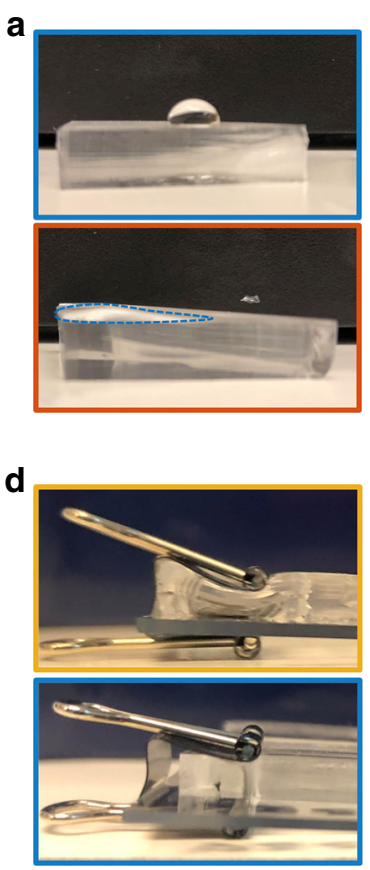

b

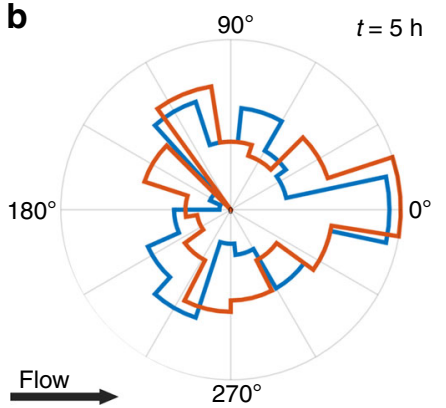

e

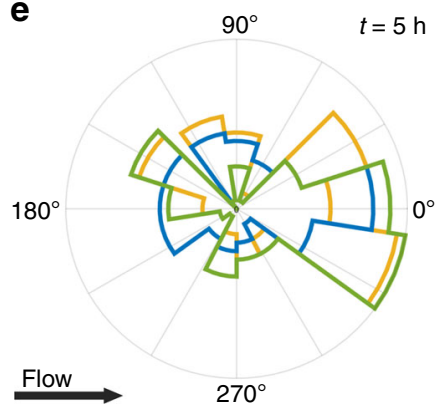

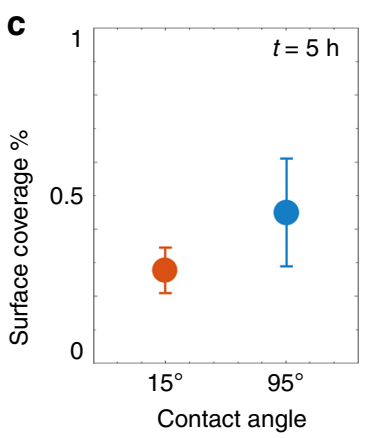

f

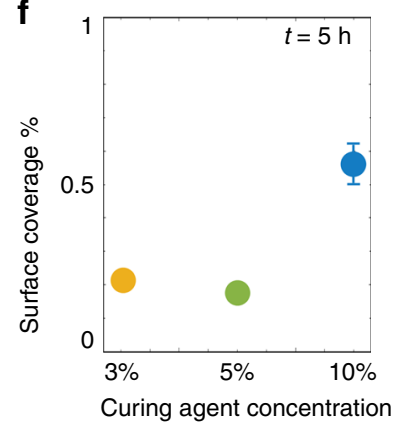

Fig. 6 Surface properties of the pillar do not affect the angular distribution of bacterial colonization. a Behavior of tryptone broth droplets on PDMS surfaces measured 5 days (upper panel; contact angle $95^{\circ} \pm 5^{\circ}$ ) and $1 \mathrm{~h}$ (lower panel; contact angle $15^{\circ} \pm 5^{\circ}$ ) after plasma treatment showing, respectively, the hydrophobic and hydrophilic nature of the two surfaces. In the lower panel, the droplet wets the surface creating a film: its borders are marked with a blue dashed line in the image for ease of visualization. b Angular distribution of the fluorescence intensity, l, of PA14 wt GFP cells attached on a hydrophobic pillar (blue) and on a hydrophilic pillar (orange), for a flow velocity at $U / V=6.6$ and a pillar diameter of $100 \mu \mathrm{m}$. c Surface coverage (measured on the upper surface of the microfluidic channel) for the hydrophobic surface (blue) and the hydrophilic surface (orange) under the same experimental conditions as b. d Slabs of PDMS containing 3\% curing agent (upper panel; Young modulus $=150 \pm 50 \mathrm{kPa}$ ) and $10 \%$ curing agent (lower panel; Young modulus $=2.25 \pm 0.25 \mathrm{MPa}$ ) undergoing compression from a binder clip in order to visualize the difference in stiffness. e Angular distribution of the fluorescence intensity, l, of PA14 wt GFP cells attached on a pillar containing 3\% (yellow), 5\% (green) and 10\% (blue) curing agent, for a flow velocity at $U / V=6.6$ and a pillar diameter of $100 \mu \mathrm{m}$. f Surface coverage (measured on the upper surface of the microfluidic channel) for PDMS containing different concentrations of curing agent under the same experimental conditions as e. Angular distributions and surface coverages were measured after $5 \mathrm{~h}$ of continuous flow. Error bars correspond to the standard error of the mean.

that the effect of different surface elements on bacterial trajectories is not independent, but rather a bacterial trajectory integrates all the effects of successive curvature elements. Fundamentally, however, observations and the model support both the existence and the leeward localization of preferential attachment on irregularly shaped surfaces.

\section{Discussion}

The role of bacterial motility in favoring contact with and thus attachment to surfaces has long been known ${ }^{50}$, and is considered an important early step in the transition from a planktonic state to the surface-associated state that initiates biofilm development. However, the role of fluid flow on this process has rarely been considered from a mechanistic viewpoint, with regard to determining how the effect of flow on the trajectories of bacteria influences surface colonization. For flat surfaces, flow has been proposed to favor surface colonization through shear trapping ${ }^{12}$. Here we focused on curved surfaces and showed two major consequences of flow. First, in the presence of flow, bacterial motility strongly enhances the attachment of bacteria to surfaces. Second, fluid flow-in concert with bacterial elongation and swimming traits (speed, tumbling rate)-determines where a cell will contact a curved surface, resulting in surface colonization patterns, and in particular leeward attachment, that are strikingly different from those expected when ignoring the effect of flow or motility. The former observation extends results, mostly theoretical, obtained for the colonization of spherical aggregates, primarily in the context of sinking particles in the ocean ${ }^{36,37}$, to cylindrical pillars of different dimensions and to a wide range of flow rates, which has particular relevance for applications ranging from filtration processes to biofouling and bioclogging. The second observation, to the best of our knowledge, has not been reported before, yet our experiments with different surface geometries, adherence properties and bacterial species indicate that the phenomenon of leeward attachment is likely to be very general.

The magnitude of fluid flow plays a fundamental role in leeward attachment. Once bacteria have been reoriented by flow, leeward attachment occurs if bacteria can swim against the flow in the wake of the pillar or other curved surfaces. When flow is too high, this cannot occur. Therefore, leeward attachment is not observed at flow velocities that are much higher than bacterial swimming speed. Here we observed leeward attachment for flow velocities as high as 13-fold the bacterial swimming speed, or $600 \mu \mathrm{m} \mathrm{s}^{-1}$ (for P. aeruginosa, which swim at $45 \mu \mathrm{m} \mathrm{s}^{-1}$ ). While the exact threshold value of flow velocity below which leeward attachment is expected depends on the bacterial swimming velocity and elongation, flow velocities of a magnitude at which leeward capture is predicted occur very frequently in natural bacterial habitats. For example, in the soil, flow velocities for groundwater can vary between 1 and $1000 \mu \mathrm{m} \mathrm{s}^{-1}$, depending on the soil type $\mathrm{e}^{51,52}$.

The process of shear-induced reorientation that leads to leeward attachment depends on two bacterial phenotypes: shape and motility in bulk. The role of these two phenotypes was already recognized in the related process of shear-induced trapping ${ }^{12}$ and 
a

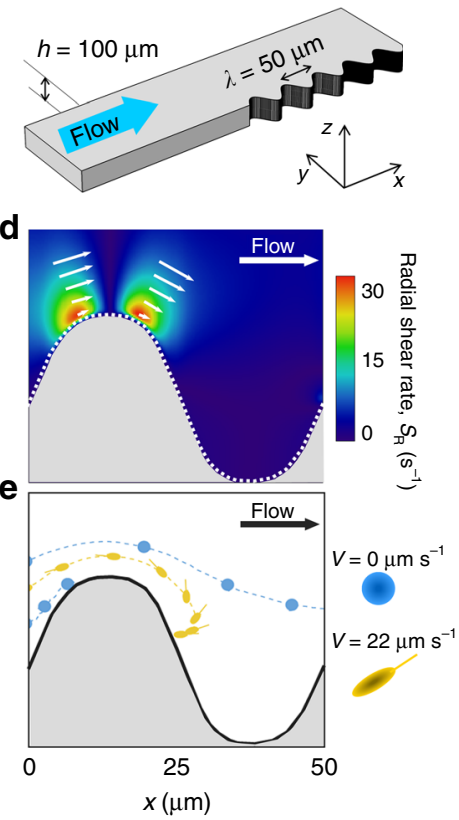

b

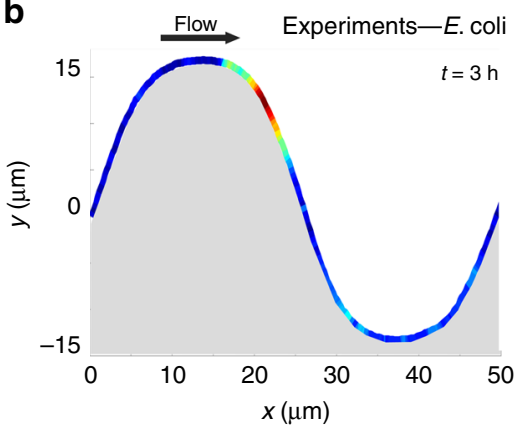

C

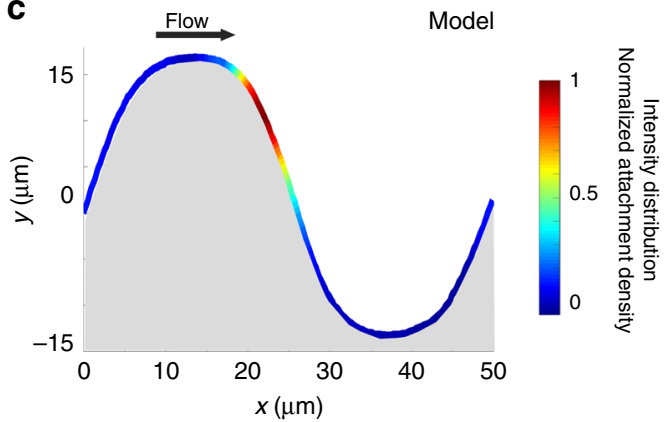

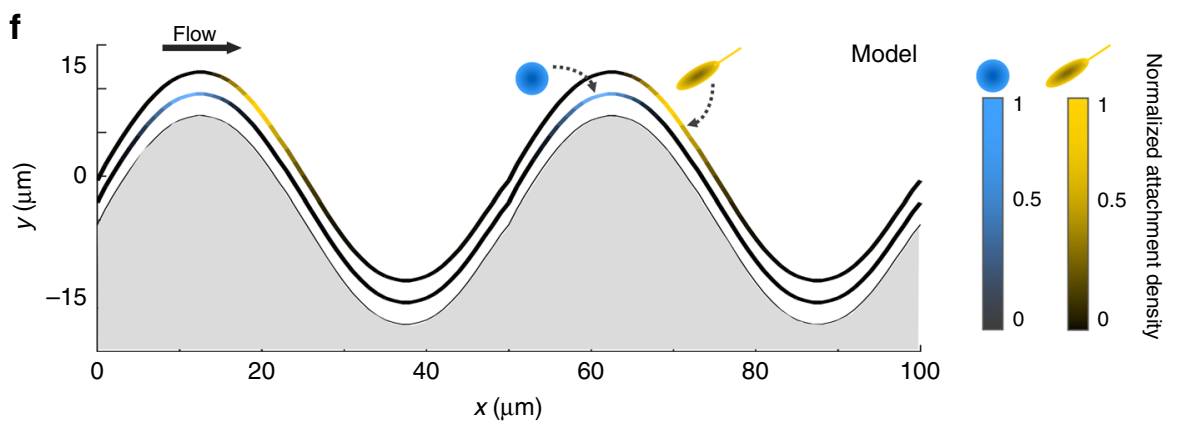

Fig. 7 Preferential leeward attachment on a sinusoidal surface by motile $\boldsymbol{E}$. coli. a Schematic of the microchannel with a sinusoidal lateral surface with wavelength $50 \mu \mathrm{m}$ and amplitude of $25 \mu \mathrm{m}$. b Distribution of the fluorescence intensity from GFP-tagged E. coli wt cells attached to the sinusoidal surface after $3 \mathrm{~h}$ of flow at $U=150 \mu \mathrm{m} \mathrm{s}^{-1}(U / V=6.9)$, averaged over 100 identical periods and normalized for the maximum intensity value. c Normalized attachment density of cells on the sinusoidal surface obtained from the model at $U=150 \mu \mathrm{m} \mathrm{s}^{-1}$. d Radial shear rate, $S_{R}$, around one period of the sinusoidal lateral wall, computed with a finite element method at $U=500 \mu \mathrm{m} \mathrm{s}^{-1}$. Superimposed arrows indicate the local velocity field. e Simulated trajectories of spherical nonmotile (blue) and of elongated motile (yellow) cells in flow around a period of the sinusoidal wall. $\mathbf{f}$ Simulated attachment density of spherical nonmotile and motile bacteria on the sinusoidal wall at $U=150 \mu \mathrm{m} \mathrm{s}^{-1}$.

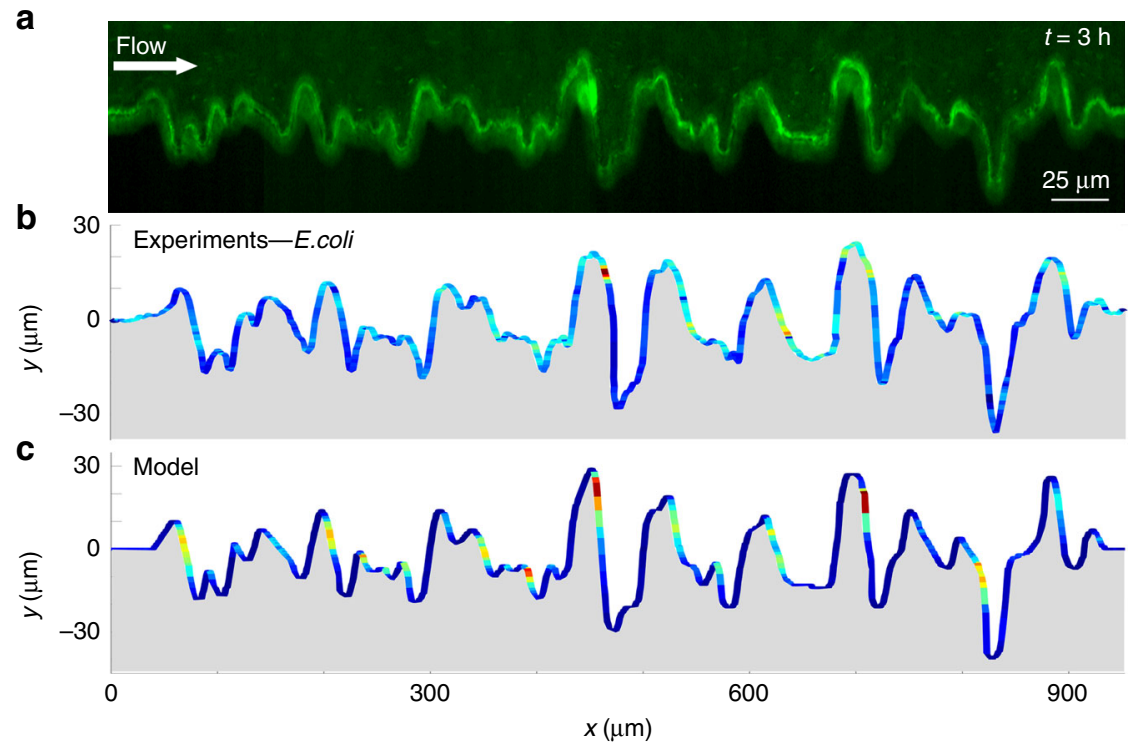

Intensity distribution, normalized attachment density

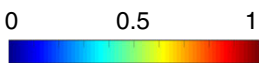

Fig. 8 Colonization of a randomly corrugated surface by E. coli. a Fluorescent image acquired at channel mid-plane of GFP-tagged E. coli wt cells attached to the lateral corrugated surface of a microfluidic channel (height $=100 \mu \mathrm{m}$ ) after $3 \mathrm{~h}$ of flow of a diluted bacterial suspension at a mean flow velocity of $U=150 \mu \mathrm{m} \mathrm{s}^{-1}$. b Intensity distribution of the fluorescent signal shown in a, normalized by its maximum value. c Normalized attachment density of cells (elongation $q=8.5$, swimming speed $V=21.6 \mu \mathrm{m} \mathrm{s}^{-1}$ ) on the corrugated surface obtained with the model at a mean flow velocity of $U=150 \mu \mathrm{m} \mathrm{s} \mathrm{s}^{-1}$ $(U / V=6.9)$. 
was here confirmed by our mathematical model for leeward attachment. A direct consequence of this dependence is a potential niche differentiation of bacterial species having different phenotypic traits: for a given surface geometry and flow velocity, bacteria with different shapes or different swimming characteristics will attach to different regions of the surface. To illustrate this, we used the model to predict the colonization of a sinusoidally corrugated surface by two different bacteria: a spherical, nonmotile bacterium (Fig. 7e, blue) and an elongated, motile bacterium (Fig. 7e, yellow). The results show that flow causes nonmotile bacteria to attach preferentially windward of apexes and motile bacteria preferentially leeward of apexes on the sinusoidal corrugation (Fig. 7f). Conversely, leeward attachment does not seem to depend on the specific surface motility strategy. For example, $P$. aeruginosa cells move on solid substrates by twitching motility using type- 4 pili ${ }^{53}$, while $E$. coli crawls on surfaces using type- 1 fimbriae ${ }^{54}$, yet both species exhibited strong leeward attachment. Moreover, once formed, the hotspots of colonization that we report do not change in shape, position and surface cell density on a time scale of hours or even upon a change in the flow direction (Supplementary Fig. 5), proving that bacteria relocation and detachment are not responsible for leeward attachment. Thus even though the optical configuration of our experimental platform does not allow inspection of bacterial behavior on the surface and consequently ruling out of the possibility that bacteria move on the surface, as observed for flat surfaces $^{45,55}$, our observations suggest that any surface redistribution of bacteria is very slow and inconsequential for the observed cell distributions. We conclude that, while other processes may still be additionally at play, the mechanism of leeward attachment proposed here and demonstrated through the good agreement between observations and simulations is the one responsible for the observed bacterial accumulations.

Since attachment sites are the seeding ground for biofilm formation, the interaction of flow and motility described here provides a mechanism for heterogeneous seeding of surfaces by bacteria with different phenotypic traits. Heterogeneity therefore can arise in a biofilm not only during development, due to spatial segregation driven by biological interactions, but can be present $\mathrm{ab}$ initio due to the physics of how bacteria encounter surfaces. Cell morphology has already been shown to be beneficial for surface colonization in flow in Caulobacter crescentus, suggesting that specific shapes can favor bacteria in different hydrodynamic conditions ${ }^{11}$. At the community level, this interplay between phenotypic traits and flow could determine long-term population dynamics, akin to the flow-induced spatial segregation of Vibrio cholerae strains based on their different adhesiveness on flat surfaces $^{56}$. Our results suggest that in environments characterized by flow, niches of bacterial colonization of surfaces may be a function of cell morphology and swimming behavior, and that the flow environment can significantly affect bacterial metapopulation dynamics by creating a feedback between flow conditions, surface topography and competition among species ${ }^{56,57}$. This preferential attachment can thus play an important role in determining the structure, adaptation and potentially the evolution of microbial communities in aqueous ecosystems ${ }^{58}$, and in medical ${ }^{24,25}$ and industrial settings $\mathrm{s}^{5,6}$.

We have shown that flow-motility interaction can favor the formation of colonization hotspots on curved surfaces. Colonization hotspots, in turn, are favorable sites for biofilm formation and quorum sensing ${ }^{59-62}$. Strong flow can repress quorum sensing, by diluting the concentration of inducer molecules, but the effect of flow is strongly quenched in sheltered regions, such as nooks and crevices ${ }^{59}$. Leeward attachment hotspots may promote quorum sensing both due to the higher local bacterial density and by the sheltered nature of the attachment location. Since antibiotic resistance and pathogenicity are behaviors mediated by quorum sensing ${ }^{61,63}$, this result highlights the potential importance of flow, motility, and surface geometry in a wide range of health-related processes, with implications for the design of filters and medical devices. Moreover, the observation that bacterial transport and attachment is largely controlled by bacterial morphology, surface topography, and flow demonstrates a previously unidentified and potentially ubiquitous interaction contributing to surface colonization in fluid environments. This knowledge, and the quantitative mechanistic model we have proposed, opens new frontiers in the possibility of controlling the colonization of surfaces by bacteria, and calls for a better understanding of the ecological and technological consequences in the many applications where the formation of biofilms is either desirable, such as in wastewater treatment plants and bioremediation systems, or to be avoided, such as artificial implants, medical devices, and desalination membranes.

\section{Methods}

Bacterial cultures. Experiments were performed using GFP-tagged $P$. aeruginosa strain PA14 wild type, flagella-deficient strain PA14 flgE and motility-deficient strain PA14 motB, and GFP-tagged E. coli strain MG1655 wild type. P. aeruginosa and E. coli solutions were prepared by inoculating $3 \mathrm{~mL}$ Tryptone Broth (TB, $10 \mathrm{~g} \mathrm{~L}^{-1}$ tryptone) from a frozen stock and incubating overnight at $37^{\circ} \mathrm{C}$, while shaking at $200 \mathrm{rpm}$. Approximately $30 \mu \mathrm{L}$ of solution was then resuspended in $3 \mathrm{~mL}$ of the same medium and incubated under the same conditions for $3 \mathrm{~h}$. To ensure a high percentage of motile bacteria in the experiments, nonmotile and dead cells were gently removed from bacterial suspensions using sterile cell culture inserts incorporating a $3-\mu \mathrm{m}$-pore-size membrane, following a procedure described before $^{12}$ (Supplementary Methods).

To investigate the impact of the medium on the bacterial distribution, experiments were also carried out using TB diluted 1:10 in an isotonic saline solution $\left(\mathrm{NaCl} 5 \mathrm{~g} \mathrm{~L}^{-1}\right)$ and $\mathrm{AB}$ minimal medium. P. aeruginosa cells were centrifuged $(1000 \times g$ for $10 \mathrm{~min})$ and then resuspended in the $\mathrm{AB}$ medium. The bacterial preparation procedures did not affect bacterial swimming speed or tumbling rate.

Microfluidic assays. To analyze surface attachment in flow around a pillar, we fabricated a microfluidic device with four channels on the same chip, each containing six pillars: two each of diameter 50, 100 and $200 \mu \mathrm{m}$ (Fig. 1a). In order to ensure that the dominant velocity gradients occurred near the pillars, we designed the microchannel with aspect ratio $H / W=0.1$ (height $H=100 \mu \mathrm{m}$; width $W=1$ $\mathrm{mm}$ ). To analyze surface attachment in flow around pillars of different wettability, we fabricated a microfluidic device with four channels on the same chip. Two channels were plasma-treated and bonded 5 days before the experiment, and two were bonded just $1 \mathrm{~h}$ before the experiment. Due to the PDMS hydrophobic recovery ${ }^{64}$, surfaces with a very different contact angle were obtained (Fig. 6a; contact angle $95^{\circ} \pm 5^{\circ}$ for the sample with 5 days of recovery time and of $15^{\circ} \pm 5^{\circ}$ for that with $1 \mathrm{~h}$ recovery, estimated from images of water droplets on the surface). To analyze surface attachment in flow around pillars of different stiffness, we fabricated a microfluidic device with six channels on the same chip. Stiffness was controlled by altering the concentration of the cross-linking agent in the PDMS solution (Fig. 6d). Pairs of channels were produced by adding a cross-linker concentration of 3,5 , and $10 \%$ (typical concentration). All the channels were cured at $80^{\circ} \mathrm{C}$ for $3 \mathrm{~h}$, bonded to glass, and then stored for $24 \mathrm{~h}$ at ambient temperature to fully polymerize. According to literature ${ }^{65,66}$, the PDMS has a Young modulus in the range of $150 \pm 50 \mathrm{kPa}$ with a concentration of $3 \%$, of $500 \pm 100 \mathrm{kPa}$ with $5 \%$, and of $2.25 \pm 0.25 \mathrm{MPa}$ with $10 \%$. To analyze surface attachment in sinusoidal and corrugated channels, we fabricated two microfluidic channels with one flat wall and the other wall having either sinusoidal features (Fig. 7a) or irregularly corrugated features (Fig. 8). Potential confounding factors stemming from cell growth, cell-cell signaling, extracellular matrix production and biological variability were minimized by focusing on early attachment $(<5 \mathrm{~h})$ and by performing experiments at different shear rates in parallel using a single cell culture. Flow was driven by a syringe pump (neMESYS $290 \mathrm{~N}$, CETONI, Germany), using flow rates in the range $Q=0.6-6$ $\mu \mathrm{L} \mathrm{min}{ }^{-1}$. Prior to use, all the microfluidic channels were washed with $2 \mathrm{~mL}$ of medium. A diluted PA14 bacteria suspension $(\mathrm{OD}<0.01$; cell concentration $<10^{6}$ cells $\mathrm{mL}^{-1}$ ) was flown for $5 \mathrm{~h}$. All experiments were performed at room temperature.

Cell imaging and tracking. All imaging was performed on an inverted microscope (Ti-Eclipse, Nikon, Japan) using a digital camera (ORCA-Flash4.0 V3 Digital CMOS camera, Hamamatsu Photonics, Japan). Bacterial trajectories (Fig. 4a), were acquired using phase-contrast microscopy (30× magnification, 200 frames per second). Bacterial attachment (Fig. 1d) was quantified using epifluorescence 
microscopy (30× magnification, 6 images per hour). All image analysis was performed in Matlab (The Mathworks) using in-house cell tracking algorithms.

Statistics and derivations. All images of bacterial attachment on pillars were taken at channel mid-depth after $5 \mathrm{~h}$ of continuous flow. The fluorescent image shown in Fig. 1d was acquired during a single experiment. The intensity distributions of the fluorescent signal shown in Fig. 2a-c were averaged over two identical pillars; the experiment was repeated three times with consistent results, but data shown are from a single realization. Data on experimental capture efficiency shown in Fig. 3a, c, on angular distributions of the fluorescence intensity shown in Fig. 5a-d, and on the normalized standard deviation $\sigma_{\theta} / \sigma_{u}$ shown in Fig. 5e was obtained from experiments repeated at least three times, and error bars correspond to the standard error of the mean. Each value was averaged over at least six identical (i.e., same diameter, same material) pillars and error bars correspond to the standard error of the mean. Sample trajectories of PA14 shown in Fig. 4a represent the longest trajectories obtained experimentally of bacteria colliding with the pillar; out of a total of $10^{3}$ trajectories recorded, 80 intercepted the pillar. Each of the angular distributions of the fluorescent intensity shown in Fig. 6 b, e was averaged over four identical pillars. The surface coverage data shown in Fig. $6 \mathrm{c}$, $\mathrm{f}$ were obtained as an average of the surface coverage measured in 12 images $(450 \mu \mathrm{m} \times 450 \mu \mathrm{m})$ of the PDMS surface, at a location $2 \mathrm{~mm}$ upstream from each pillar and on the upper PDMS surface of the microchannel; error bars correspond to the standard error of the mean. The intensity distribution of the fluorescence intensity shown in Fig. $7 \mathrm{~b}$ was averaged over 100 identical periods and normalized by the maximum intensity value. The fluorescent image shown in Fig. 8a was acquired during a single experiment.

Quantification of the capture efficiency. In the model results, the capture efficiency $\eta_{C}{ }^{\text {mod }}$ was calculated, according to its definition, as the fraction of bacteria removed from the volume of water subtended by the pillar's cross-section ${ }^{31,35}$. In the experiments, the capture efficiency $\eta_{C}{ }^{\text {exp }}$ was obtained (see Eqn. (7) in ref. 35) by dividing the bacterial capture rate by the flux of particles encountering the cylinder, $F=P U d_{\mathrm{P}} l_{\mathrm{P}}$, where $P$ is the particle concentration in the flowing fluid and $l_{\mathrm{P}}$ is the height of the cylinder (here set to 1 , since we are considering a $2 \mathrm{D}$ plan at channel mid-depth). Capture rate is assumed proportional to bacterial attachment, where the latter was measured in terms of fluorescent intensity ${ }^{27}, I_{\mathrm{IN}}$, of all pixels integrated over a $5-\mu \mathrm{m}$-thick annulus around the perimeter of the pillar. We thus define the experimental capture efficiency as $\eta_{\mathrm{C}}{ }^{\exp }=I_{\mathrm{IN}} /\left(P U d_{\mathrm{P}}\right)$.

Numerical simulations. The mathematical model is based on first computing the $3 \mathrm{D}$ velocity field inside the microchannel (with COMSOL Multiphysics) and then using it to simulate the transport of individual bacteria, for the same geometry and flow conditions as in the experiments. We modeled bacteria as prolate ellipsoids with an effective aspect ratio $q$, which accounts for the combined hydrodynamic resistance of cell body and flagellar bundle, and swimming speed $V$ directed along the cells' long axis. A cell's equations of motion in the 2D flow that occurs in the experimental observation plane (i.e., channel mid-depth; Fig. 1b) are

$$
\begin{gathered}
\dot{x}=\frac{\partial \psi}{\partial x}+V \cos \theta \\
\dot{y}=-\frac{\partial \psi}{\partial x}+V \sin \theta \\
\dot{\theta}=\frac{q^{2}-1}{q^{2}+1}\left[\frac{1}{2}\left(\frac{\partial^{2} \psi}{\partial y^{2}}-\frac{\partial^{2} \psi}{\partial x^{2}}\right) \cos 2 \theta-\frac{\partial^{2} \psi}{\partial x \partial y} \sin 2 \theta\right]-\frac{1}{2}\left(\frac{\partial^{2} \psi}{\partial x^{2}}+\frac{\partial^{2} \psi}{\partial y^{2}}\right)+\xi_{\mathrm{R}},
\end{gathered}
$$

where $\psi$ is the stream function and $\xi_{\mathrm{R}}$ is the rotational noise represented as a Gaussian-distributed angular velocity with mean zero and variance $2 D_{\mathrm{R}} / \Delta t$, where $\Delta t$ is the elapsed time and $D_{\mathrm{R}}$ is the cell's effective rotational diffusivity. The equations of motion were integrated numerically for up to $5 \times 10^{5}$ bacteria using a fourth-order Runge-Kutta scheme implemented in Matlab (The MathWorks). The number of simulated bacteria is comparable to the number of bacteria $\left(10^{5}-10^{6}\right)$ crossing a $500 \mu \mathrm{m}$-wide section of the channel, during a $5 \mathrm{~h}$ experiment, with a cell concentration of $10^{6}$ cells $\mathrm{mL}^{-1}$ and for an average flow velocity in the range of $150 \mu \mathrm{m} \mathrm{s}^{-1}<U<2 \mathrm{~mm} \mathrm{~s}^{-1}$. The swimming speed and rotational diffusivity were measured experimentally (Supplementary Fig. 1), while the effective aspect ratio, $q$, was computed from resistive force theory (Supplementary Methods). For $P$. aeruginosa we used $q=9.4, V=45 \mu \mathrm{m} \mathrm{s}^{-1}$, and $D_{\mathrm{R}}=1.4 \mathrm{rad}^{2} \mathrm{~s}^{-1}$ (Supplementary Fig. 1). For $E$. coli we used $q=8.5, V=21.6 \mu \mathrm{m} \mathrm{s} \mathrm{s}^{-1}$, and $D_{\mathrm{R}}=0.32 \mathrm{rad}^{2} \mathrm{~s}^{-1}$ (Supplementary Methods). For spherical nonmotile bacteria (Fig. 7e, f) we used $q=1, V=D_{\mathrm{R}}=0$. In the simulations, the swimmers start $400 \mu \mathrm{m}$ upstream of the pillar (i.e., the beginning of the flow field) with random positions across the channel width $(y)$ and random orientations $(\theta)$. Each time a swimmer contacts the pillar surface or exits the flow field it is reinjected with prescribed initial conditions. For contact between bacteria and surfaces, we used a "perfectly sticking" condition, i.e., any collision between the simulated trajectory and the boundary of the pillar or of the corrugation is considered as the bacterium irreversibly attaching to the surface. This condition, which neglects any hydrodynamic or specific interaction between the cell and the surface of the pillar, represents a good approximation for the surface attachment of biofilm-forming bacteria, such P. aeruginosa and E. coli.

\section{Data availability}

The data that support the findings of this study are available from the corresponding authors upon request.

Received: 19 June 2019; Accepted: 15 May 2020;

Published online: 05 June 2020

\section{References}

1. Flemming, H.-C. et al. Biofilms: an emergent form of bacterial life. Nat. Rev Microbiol. 14, 563-575 (2016).

2. Hall-stoodley, L., Costerton, J. W. \& Stoodley, P. Bacterial biofilms: from the natural environment to infectious diseases. Nat. Rev. Microbiol. 2, 95-108 (2004).

3. Stewart, P. S. \& Costerton, J. W. Antibiotic resistance of bacteria in biofilms Lancet 358, 135-138 (2001).

4. Lane, M. C. et al. Role of motility in the colonization of uropathogenic Escherichia coli in the urinary tract. Infect. Immun. 73, 7644-7656 (2005).

5. Flemming, H. C. Biofouling in water systems-Cases, causes and countermeasures. Appl. Microbiol. Biotechnol. 59, 629-640 (2002).

6. Baker, J. S. \& Dudley, L. Y. Biofouling in membrane systems-A review. Desalinization 118, 81-90 (1998).

7. Kannan, A., Yang, Z., Kim, M. K., Stone, H. A. \& Siryaporn, A. Dynamic switching enables efficient bacterial colonization in flow. Proc. Natl Acad. Sci. USA 115, 5438-5443 (2018)

8. Shen, Y., Siryaporn, A., Lecuyer, S., Gitai, Z. \& Stone, H. A. Flow directs surface-attached bacteria to twitch upstream. Biophys. J. 103, 146-151 (2012).

9. Lecuyer, S. et al. Shear stress increases the residence time of adhesion of Pseudomonas aeruginosa. Biophys. J. 100, 341-350 (2011).

10. Persat, A., Inclan, Y. F., Engel, J. N., Stone, H. A. \& Gitai, Z. Type IV pili mechanochemically regulate virulence factors in Pseudomonas aeruginosa. Proc. Natl Acad. Sci. USA 112, 7563-7568 (2015).

11. Persat, A., Stone, H. A. \& Gitai, Z. The curved shape of Caulobacter crescentus enhances surface colonization in flow. Nat. Commun. 5, 1-9 (2014).

12. Rusconi, R., Guasto, J. S. \& Stocker, R. Bacterial transport suppressed by fluid shear. Nat. Phys. 10, 2-7 (2014).

13. Ezhilan, B. \& Saintillan, D. Transport of a dilute active suspension in pressuredriven channel flow. J. Fluid Mech. 777, 482-522 (2015)

14. Miño, G. L. et al. E. coli accumulation behind an obstacle. Adv. Microbiol 8 , 451-464 (2018).

15. Altshuler, E. et al. Flow-controlled densification and anomalous dispersion of E. coli through a constriction. Soft Matter 9, 1864-1870 (2013).

16. Rusconi, R., Lecuyer, S., Guglielmini, L. \& Stone, H. A. Laminar flow around corners triggers the formation of biofilm streamers. J. R. Soc. Interface 7 , 1293-1299 (2010)

17. Rusconi, R., Lecuyer, S., Autrusson, N., Guglielmini, L. \& Stone, H. A. Secondary flow as a mechanism for the formation of biofilm streamers. Biophys. J. 100, 1392-1399 (2011)

18. Autrusson, N., Guglielmini, L., Lecuyer, S., Rusconi, R. \& Stone, H. A. The shape of an elastic filament in a two-dimensional corner flow. Phys. Fluids 23, 063602 (2012)

19. Liu, Y. et al. Role of collector alternating charged patches on transport of Cryptosporidium parvum oocysts in a patchwise charged heterogeneous micromodel. Environ. Sci. Technol. 47, 2670-2678 (2013).

20. Kim, H. N., Walker, S. L. \& Bradford, S. A. Coupled factors influencing the transport and retention of Cryptosporidium parvum oocysts in saturated porous media. Water Res. 44, 1213-1223 (2010).

21. Le Borgne, T., Bolster, D., Dentz, M., De Anna, P. \& Tartakovsky, A. Effective pore-scale dispersion upscaling with a correlated continuous time random walk approach. Water Resour. Res. 47, 1-11 (2011).

22. Van de Moortele, T., Goerke, U., Wendt, C. H. \& Coletti, F. Airway morphology and inspiratory flow features in the early stages of Chronic Obstructive Pulmonary Disease. Clin. Biomech. 66, 60-65 (2019).

23. Vahidi, B., Fatouraee, N., Imanparast, A. \& Moghadam, A. N. A Mathematica simulation of the ureter: effects of the model parameters on ureteral pressure/ flow relations. J. Biomech. Eng. 133, 031004 (2010).

24. Kim, H. J., Li, H., Collins, J. J. \& Ingber, D. E. Contributions of microbiome and mechanical deformation to intestinal bacterial overgrowth and inflammation in a human gut-on-a-chip. Proc. Natl Acad. Sci. USA 113 E7-E15 (2016).

25. Nawroth, J. C. et al. Motile cilia create fluid-mechanical microhabitats for the active recruitment of the host microbiome. Proc. Natl Acad. Sci. USA 114, 9510-9516 (2017)

26. Shapiro, O. H. et al. Vortical ciliary flows actively enhance mass transport in reef corals. Proc. Natl Acad. Sci. USA 111, 13391-13396 (2014).

27. Sipos, O., Nagy, K., Di Leonardo, R. \& Galajda, P. Hydrodynamic trapping of swimming bacteria by convex walls. Phys. Rev. Lett. 114, 258104 (2015) 
28. Mok, R., Dunkel, J. \& Kantsler, V. Geometric control of bacterial surface accumulation. Phys. Rev. E 99, 052607 (2019).

29. Desai, N., Shaik, V. A. \& Ardekani, A. M. Hydrodynamic interaction enhances colonization of sinking nutrient sources by motile microorganisms. Front. Microbiol. 10, 289 (2019).

30. Spielman, L. A. Particle capture from low-speed laminar flows. Annu. Rev. Fluid Mech. 9, 297-319 (1977)

31. Friedlander, S. K. Particle diffusion in low-speed flows. J. Colloid Interface Sci. 23, 157-164 (1967).

32. Friedlander, S. K. Mass and heat transfer to single spheres and cylinders at low Reynolds numbers. Aiche J. 3, 43-48 (1957).

33. Natanson, G. L. Diffusive deposition of aerosols on a cylinder in a flow in the case of small capture coefficients. Dokl. Akad. Nauk SSSR 112, 100-103 (1957).

34. Espinosa-Gayosso, A., Ghisalberti, M., Ivey, G. N. \& Jones, N. L. Particle capture and low-Reynolds-number flow around a circular cylinder. J. Fluid Mech. 710, 362-378 (2012).

35. Palmer, M. R., Nepf, H. M. \& Pettersson, T. J. R. Accumulation and removal in aquatic systems. Limnol. Oceanogr. 49, 76-85 (2004).

36. Kiørboe, T., Grossart, H. P., Ploug, H. \& Tang, K. Mechanisms and rates of colonisation of sinking aggregates. Appl. Environ. Microbiol. 68, 3996-4006 (2002).

37. Kiørboe, T. \& Jackson, G. A. Marine snow, organic solute plumes, and optimal chemosensory behavior of bacteria. Limnol. Oceanogr. 46, 1309-1318 (2001).

38. Lambert, B. S., Fernandez, V. I. \& Stocker, R. Motility drives bacterial encounter with particles responsible for carbon export throughout the ocean. Limnol. Oceanogr. 4, 113-118 (2019).

39. De Anna, P. et al. Mixing and reaction kinetics in porous media: An experimental pore scale quantification. Environ. Sci. Technol. 48, 508-516 (2014).

40. Humphries, S. Filter feeders and plankton increase particle encounter rates through flow regime control. Proc. Natl Acad. Sci. USA 106, 7882-7887 (2009).

41. Peruzzo, P., Defina, A., Nepf, H. M. \& Stocker, R. Capillary interception of floating particles by surface-piercing vegetation. Phys. Rev. Lett. 111, 164501 (2013).

42. Lee, G. S. et al. Blood flow shapes intravascular pillar geometry in the chick chorioallantoic membrane. J. Angiogenes. Res. 2, 1-9 (2010).

43. Howse, J. R. et al. Self-motile colloidal particles: from directed propulsion to random walk. Phys. Rev. Lett. 99, 8-11 (2007).

44. Guasto, J. S., Rusconi, R. \& Stocker, R. Fluid mechanics of planktonic microorganisms. Annu. Rev. Fluid Mech. 44, 373-400 (2012).

45. Hill, J., Kalkanci, O., McMurry, J. L. \& Koser, H. Hydrodynamic surface interactions enable Escherichia coli to seek efficient routes to swim upstream. Phys. Rev. Lett. 98, 1-4 (2007).

46. Mathijssen, A. J. T. M., Shendruk, T. N., Yeomans, J. M. \& Doostmohammadi, A. Upstream swimming in microbiological flows. Phys. Rev. Lett. 116, 028104 (2016).

47. Jeffrey, G. B. The motion of ellipsoidal particles immersed in a viscous fluid. Proc. R. Soc. A 102, 161 (1922).

48. Barry, M. T., Rusconi, R., Guasto, J. S. \& Stocker, R. Shear-induced orientational dynamics and spatial heterogeneity in suspensions of motile phytoplankton. J. R. Soc. Interface 12, 20150791 (2015).

49. Miller, M. J., Wilson, L. A. \& Ahearn, D. G. Adherence of Pseudomonas aeruginosa to rigid gas-permeable contact lenses. Arch. Ophthalmol. 109, 1447-1448 (1991).

50. O'Toole, G. A. \& Kolter, R. Flagellar and twitching motility are necessary for Pseudomonas aeruginosa biofilm development. Mol. Microbiol. 30, 295-304 (1998).

51. Taylor, R., Cronin, A., Pedley, S., Barker, J. \& Atkinson, T. The implications of groundwater velocity variations on microbial transport and wellhead protection-Review of field evidence. FEMS Microbiol. Ecol. 49, 17-26 (2004).

52. Mackay, D. M., Roberts, P. V. \& Cherry, J. A. Transport of organic contaminants in groundwater. Environ. Sci. Technol. 19, 384-392 (1985).

53. Burrows, L. L. Pseudomonas aeruginosa twitching motility: type IV pili in action. Annu. Rev. Microbiol. 66, 493-520 (2012).

54. Lane, M. C., Simms, A. N. \& Mobley, H. L. T. Complex interplay between type 1 fimbrial expression and flagellum-mediated motility of uropathogenic Escherichia coli. J. Bacteriol. 189, 5523-5533 (2007).

55. Mathijssen, A. J. T. M. et al. Oscillatory surface rheotaxis of swimming E. coli bacteria. Nat. Commun. 10, 7-9 (2019).

56. Martínez-García, R., Nadell, C. D., Hartmann, R., Drescher, K. \& Bonachela, J. A. Cell adhesion and fluid flow jointly initiate genotype spatial distribution in biofilms. PLoS Comput. Biol. 14, 1-19 (2018).

57. Nadell, C. D., Ricaurte, D., Yan, J., Drescher, K. \& Bassler, B. L. Flow environment and matrix structure interact to determine spatial competition in Pseudomonas aeruginosa biofilms. Elife 6, 1-14 (2017).
58. Zhou, J. et al. Stochasticity, succession, and environmental perturbations in a fluidic ecosystem. Proc. Natl Acad. Sci. USA 111, E836-E845 (2014).

59. Kim, M. K., Ingremeau, F., Zhao, A., Bassler, B. L. \& Stone, H. A. Local and global consequences of flow on bacterial quorum sensing. Nat. Microbiol 1, 15005 (2016).

60. Siryaporn, A., Kuchma, S. L., O’Toole, G. A. \& Gitai, Z. Surface attachment induces Pseudomonas aeruginosa virulence. Proc. Natl Acad. Sci. USA 111, 16860-16865 (2014)

61. Papenfort, K. \& Bassler, B. L. Quorum sensing signal-response systems in Gram-negative bacteria. Nat. Rev. Microbiol. 14, 576-588 (2016).

62. Kirisits, M. J. et al. Influence of the hydrodynamic environment on quorum sensing in Pseudomonas aeruginosa biofilms. J. Bacteriol. 189, 8357-8360 (2007).

63. Hentzer, M. et al. Attenuation of Pseudomonas aeruginosa virulence by quorum sensing inhibitors. EMBO J. 22, 3803-3815 (2003).

64. Fritz, J. L. \& Owen, M. J. Hydrophobic recovery of plasma-treated polydimethylsiloxane. J. Adhes. 54, 33-45 (1995).

65. Song, F. \& Ren, D. Stiffness of cross-linked poly(dimethylsiloxane) affects bacterial adhesion and antibiotic susceptibility of attached cells. Langmuir $\mathbf{3 0}$ 10354-10362 (2014).

66. Fuard, D., Tzvetkova-Chevolleau, T., Decossas, S., Tracqui, P. \& Schiavone, P. Optimization of poly-di-methyl-siloxane (PDMS) substrates for studying cellular adhesion and motility. Microelectron. Eng. 85, 1289-1293 (2008).

\section{Acknowledgements}

We acknowledge support from an ETH Fellowship and the SNSF PRIMA Grant 179834 (to E.S), from Gordon and Betty Moore Foundation Marine Microbiology Initiative Investigator Award GBMF3783 (to R.S.), from Simons Foundation Grant 542395 (to R.S.) as part of the Principles of Microbial Ecosystems Collaborative (PriME) and from the Italian Ministry of Health - Fondi 5x1000 Ricerca Sanitaria (to R.R.).

\section{Author contributions}

E.S., R.R., G.L.M., V.K., and R.S. designed research. E.S. and R.R performed experiments on pillars, simulations for all geometries, and analyzed all the data. G.L.M. performed experiments on sinusoidal and wavy channels. A.V. and L.E. modified Pseudomonas aeruginosa strains. E.S., R.R., and R.S. wrote the manuscript and all authors edited and commented on the manuscript.

\section{Competing interests}

The authors declare no competing interests.

\section{Additional information}

Supplementary information is available for this paper at https://doi.org/10.1038/s41467 020-16620-y.

Correspondence and requests for materials should be addressed to E.S., R.R. or R.S.

Peer review information Nature Communications thanks the anonymous reviewer(s) for their contribution to the peer review of this work.

Reprints and permission information is available at http://www.nature.com/reprints

Publisher's note Springer Nature remains neutral with regard to jurisdictional claims in published maps and institutional affiliations.

Open Access This article is licensed under a Creative Commons Attribution 4.0 International License, which permits use, sharing, adaptation, distribution and reproduction in any medium or format, as long as you give appropriate credit to the original author(s) and the source, provide a link to the Creative Commons license, and indicate if changes were made. The images or other third party material in this article are included in the article's Creative Commons license, unless indicated otherwise in a credit line to the material. If material is not included in the article's Creative Commons license and your intended use is not permitted by statutory regulation or exceeds the permitted use, you will need to obtain permission directly from the copyright holder. To view a copy of this license, visit http://creativecommons.org/ licenses/by/4.0/

(C) The Author(s) 2020 